\title{
Substance abuse, drug addiction and the role of primary health care amid the COVID-19 pandemic
}

\author{
Mustafa Elsaied Esmail ${ }^{1 *}$, Majed Diaa Mosly ${ }^{1}$, Abdulaziz Abdullah Alghamdi², \\ Alalaa Hussain Hakami ${ }^{3}$, Ahmed Ali Majrashi ${ }^{4}$, Safwan Shaker Abdulrahim ${ }^{5}$, \\ Areej Mohammed Alnashry ${ }^{6}$, Anwar Saeed Alalsaba ${ }^{7}$, Baneen Abdullah Akakah ${ }^{7}$, \\ Khaled Abdullah Alshahrani ${ }^{8}$, Nawaf Hatem Salamah ${ }^{5}$
}

\author{
${ }^{1}$ Department of Psychiatry, Al Amal Mental Health Complex, Jeddah, Saudi Arabia \\ ${ }^{2}$ College of Medicine, King Abdulaziz University, Jeddah, Saudi Arabia \\ ${ }^{3}$ Department of Emergency Medicine, Prince Mohammed Bin Nasser Hospital, Jazan, Saudi Arabia \\ ${ }^{4}$ Department of Psychiatry, Eradah Mental Health Hospital, Abha, Saudi Arabia \\ ${ }^{5}$ College of Medicine, University of Jeddah, Jeddah, Saudi Arabia \\ ${ }^{6}$ Department of Psychiatry, Eradah Al Amal Mental Health Complex, Jeddah, Saudi Arabia \\ ${ }^{7}$ Department of Psychiatry, Eradah Al Amal Mental Health Complex, Dammam, Saudi Arabia \\ ${ }^{8}$ General Administration of Medical Services in Public Security, Health Center in the Great Holy Mosque of Mecca, \\ Mecca, Saudi Arabia
}

Received: 06 November 2021

Accepted: 22 November 2021

*Correspondence:

Dr. Mustafa Elsaied Esmail,

E-mail: Muustafa8011@yahoo.com

Copyright: (C) the author(s), publisher and licensee Medip Academy. This is an open-access article distributed under the terms of the Creative Commons Attribution Non-Commercial License, which permits unrestricted non-commercial use, distribution, and reproduction in any medium, provided the original work is properly cited.

\begin{abstract}
The definition of substance abuse is the use of certain types of drugs such as alcohol, cannabis, hash, cocaine and others that have serious side effects and clinical complications. There is a significant difference between substance abuse and the term addiction. The significant difference between substance abuse and addiction is that drug addiction or substance addiction is classified as a chronic disease controlling the physical and mental ability of the individual to unpleasant force to use specific drugs such as cocaine or else. The literature has a positive correlation between the COVID-19 pandemic and illegal drug abuse in substance abuse or addiction. COVID-19 caused emotional emptiness for many people around the world, in addition to anxiety and depression. The particular group of people started addicting to specific drugs to induce temporary happiness due to the unpleasant events they had from the pandemic. This article aimed to review the challenges of substance abuse and drug abuse in the COVID-19 pandemic and the role of public health in addition to primary care facilities against this phenomenon. To our knowledge, this is the first review providing a complex review about this issue since the beginning of COVID-19.
\end{abstract}

Keywords: Substance abuse, Drug addiction, COVID-19, Primary care

\section{INTRODUCTION}

The definition of substance abuse is the use of certain types of drugs such as alcohol, cannabis, amphetamine, methamphetamine, cocaine and others that have serious side effects and clinical complications. There is a significant difference between substance abuse and the term addiction. ${ }^{1}$ The significant difference between substance abuse and addiction is that drug addiction or substance addiction is classified as a chronic disease controlling the physical and mental ability of the individual to unpleasant force to use specific drugs such as amphetamine and cannabis. If the individual attempts to resist and not take the substance, significant physical 
and mental changes occur, while substance abuse does not. ${ }^{2}$ So, substance abuse can be considered an early phase before the addiction, amid the emergence of the COVID-19 pandemic in March 2020. The world has been suffering from isolation and lockdown in different places all over the world. No specific country or region was excluded from those unfortunate events. ${ }^{3}$ Among the public health problems that proliferated during the lockdown of COVID-19 and isolation is substance abuse disorders. Substance abuse disorders are a group of psychiatric diseases characterized by principal affection of the central nervous system either physically, mentally or emotionally due to frequent use of illegal substances or drugs. Substance abuse disorders are considered significant challenges for public health and global health sectors. ${ }^{4}$ The incidence of substance abuse disorders ranges from $1-5 \%$ according to several factors such as socioeconomic status, geographical location, the widespread of substances and the affordability to access specific drugs. ${ }^{5}$ The literature had a positive correlation between the COVID-19 pandemic and illegal drug abuse in substance abuse or addiction. COVID-19 caused emotional emptiness for many people around the world, in addition to anxiety and depression. The particular group of people started addicting to specific drugs to induce temporary happiness due to the unpleasant events they had from the pandemic. ${ }^{6}$ However, this has emerged as a pandemic over the pandemic for several reasons. At first, the incidence of substance abuse and illegal drug addiction has been counting for $10 \%$ of the overall population of the United States of America. The lowest incidence was $2.5 \%$ for certain drugs, while the highest was for alcohol which counted for $12 \%$. $^{7}$

Secondly, the use of illegal drugs has been linked to the human body's decreased immunological system, which implicates repeated infections easily compared to people who do not use drugs. Therefore, the effect of the SARSCoV-2 virus, which was behind the COVID-19 pandemic, will be synergistic compared to the average population, except for chronic disease patients and geriatrics. ${ }^{8}$ So, we get that the usage of illegal drugs, especially amid the COVID-19 pandemic, will result in worse results than the infections in everyday life before the era of the COVID19 pandemic, which makes what is called a pandemic philosophically over the pandemic. The role of screening and prevention of those illegal substances was mainly coordinated by the community medicine and public health sector. Therefore, organized advisory campaigns should be planned to fight this issue. This article aimed to review the challenges of substance abuse and drug abuse in the COVID-19 pandemic and the role of public health in addition to primary care facilities against this phenomenon. To our knowledge, this was the first review providing a complex review about this issue since the beginning of COVID-19.

\section{METHODS}

This literature review was based on an extensive literature search in Medline, Cochrane and EMBASE databases which was performed on 25 October 2021 using the medical subject headings (MeSH) or a combination of all possible related terms, according to the database. To avoid missing potential studies, a further manual search for papers was done through Google Scholar while the reference lists of the initially included papers. Papers discussing substance abuse, drug addiction and the role of primary health care amid the COVID-19 pandemic were screened for useful information. No limitations were posed on date, language, age of participants or publication type.

\section{DISCUSSION}

\section{Alcohol use and COVID-19}

In the middle of 2020, some rumors have been emerged claiming that drinking alcohol was an effective preventive solution for COVID-19 infection. ${ }^{9}$ Those rumors were reported from different countries around the world and not specified to a particular geographical area such as Europe and Eastern European countries. ${ }^{9}$ In March 2020, about 200 individuals in Iran died from alcoholism overdose. With the continuity of the pandemic, many people abused alcohol on a daily basis and it became popular compared to the proper use in the era before COVID-19. ${ }^{10}$ In Thailand, it was reported that alcohol sales have increased since the beginning of COVID-19 as people believe that it was an effective treatment for viruses comparing it with hand sanitizer usage for disease prevention. ${ }^{11}$ In another report, an Indian alcohol treatment center located in Mumbai city, a case was admitted due to alcohol toxicity. The history of the patient included drinking hand sanitizer as an antagonist for the SARS-CoV-2 virus. Unfortunately, the patient developed a severe skin rash and an inflammatory reaction. ${ }^{12}$ The usage of alcohol was different and not specified to one type nor one way. The variety of differences was due to social status, economic status, behavior and beliefs, religious beliefs and other health status-related conditions. However, the chronic usage of alcohol has been linked to decrease immunity status to defend against a variety of bacterial and viral infections. In addition, it predispose to other systematic diseases such as alcoholic fatty liver, Alzheimer and several issues in the gastrointestinal tract. ${ }^{13}$

\section{Tobacco abuse and COVID-19}

In the era of the COVID-19 pandemic, the complex issue of smoking and the threat of severe respiratory system infections were considered among the top burdens. Most sectors focus on tobacco safety to decrease the rate of non-communicable, cardiovascular and most cancerassociated mortalities. Electronic cigarettes advertising focused on saving billions of lives that might otherwise 
be misplaced due to this correlated issue due to tobacco abuse. ${ }^{14}$

In South Africa and India, the sale of tobacco products had been banned during the COVID-19 pandemic at the peak of the pandemic emergence. This ban was suitable and was supported via evidence of harm because the combined effect of tobacco use with COVID-19 was fatal. The current evidence reported that there was a strong relationship between respiratory system health and tobacco use. It was also found that tobacco use was linked to increased tuberculosis infection rates compared to nontobacco users. ${ }^{14}$ The literature reported that tobacco users, including all forms of smoking, were more likely to develop pneumococcal infections than ordinary healthy people who did not use any forms of smoking. Some theories link between the increased risk of COVID-19 infection and ACE2 receptors in the lung. Claiming that the overexpression and regulation of ACE2 receptors were being manifested with tobacco users and smokers compared with non-tobacco users and non-smokers, with exclusion with individuals with chronic diseases such as pulmonary fibrosis and interstitial lung disease. ${ }^{14}$ Nonetheless, the COVID-19 pandemic made individuals under more stress of job loss, lockdown and other social limitation. Therefore, tobacco abuse was seen to surge during the peak era of the pandemic.

\section{Opioids and COVID-19}

Drug overdose in the United States of America rose in 2019, although it declined slightly from 2017 to 2018. This increase was primarily driven by illegal fentanyl. ${ }^{15}$ The opioid-epidemic had been exacerbated by increased use of methamphetamine in combination with opioids, especially in 2019. It was possible that the emergence of coronavirus 2019 (COVID-19) and subsequent disruptions to healthcare systems and social security networks combined with social and economic pressures exacerbated the opioid epidemic, not only within the United States, but also around the world. Several reports and documentation from different media sources suggested that the opioid-related overdose endemic was rising. However, the lack of real-time national reporting of death-related deaths hindered the ability to verify these documentaries and reports. ${ }^{15}$

Two studies by Wainwright et al and Ochalek et al endorsed that drug use and opioid overdose throughout the COVID-19 length was increased in the following months, according to the media reports. ${ }^{16,17}$ There had been a failure over the previous decades since to offer an extensive plan for management and treatment for opioid addiction and illegal trade. Despite evidence of long-time period efficacy, the burden COVID-19 had placed on the United States of America the quality of the care for the overall infrastructure within the country capacities and capabilities decreased. Given this, like with other factors such as racial-affection, social correlations, several economic disparities in opioid overdose and COVID-19- related contamination, the advent of regulations by selfimplication will not be enough to mitigate the impact of the COVID-19 pandemic and the association with opioids distribution. It was vital to find out the unusual way to translate those guidelines into remedy practices, enlarge infrastructure and address the broader social structures and health systems that create inequalities in gaining the ability to access the best care. Even though COVID-19 made it harder for drug distribution during lockdown, evidence suggested that those with access to such drugs were more likely to suffer from opioid addiction compared to those who did not have access.

\section{Cannabis and COVID-19}

The current evidence suggested that cannabis has an antiinflammatory action in cases of COVID-19 infection, decreasing and limiting the activity of the viral infection within the immunity system of the human body. ${ }^{18}$ This was suggested to be among the ability of cannabis to regulate up the SARS-CoV-2 receptors within the body, especially the lungs. Cannabis was shown to have an influential role as an anti-inflammatory mediator by mitigating the cytokines reactions within the infectious reactions within the body. ${ }^{19}$ The hypothesis of using cannabis as a supportive treatment for COVID-19 treatment might be promising. However, there were several side effects of using cannabis and its products for COVID-19 patients. At first, as a public health problem, the widespread of cannabis and hashish as a drug of choice for a pandemic would open doors for illegal trades for cannabis and its derivatives; predicting that the abusive use for it might significantly increase compared to the previous conditions as in the era before the pandemic. Secondly, at the moment, there were not enough confirmatory trials about the safety and feasibility of using cannabis for COVID-19 as an option. All possibilities should be studied before attempting to approve cannabis as an acceptable drug within COVID19 therapeutic protocols. ${ }^{20}$

\section{Role of primary care}

One of the most common and uncommon troubles visible via number one care physicians was substance abuse. As a result of accelerated morbidity and mortality, loss of productivity and expanded health care charges, substance abuse in our society remained a considerable public health problem. Primary care physicians were inside the vanguard of hospital therapy and as a result, were in a unique place to pick out problems in this vicinity. Even though the superiority of substance abuse problems in number one care patients may be very excessive, medical doctors, due to a lack of proper schooling and doubts approximately the effectiveness of treatment, sense unprepared to deal with substance abuse issues within the workplace. Diagnosis of substance abuse can be made thru an aggregate of history, questionnaires, physical examinations and chemical assessments. Primary care physicians were appropriately located to treat drug 
abusers with records and access ethics, social offerings and pharmacologic techniques. Combining substance abuse disorders with other scientific and psychiatric comorbidities makes treating substance abuse problems very crucial in inpatient care. ${ }^{21}$

To make it clear, for primary care caregivers, substance abuse was an important thing to be addressed inside the entire patient's care. In its dating with other fitness conditions, the recognition and use of medication are essential to improving the general health of sufferers. Analysis and anticipated guidance throughout preventive visits may also tell sufferers of substance abuse dangers, and remedy plans may encompass strategies to address those issues early. With an abundance of diagnostic equipment, understanding of the substances used and sources and remedy strategies, primary care physicians were nicely equipped to assist their patients. ${ }^{21}$

\section{CONCLUSION}

The problems of substance abuse and drug addiction generally, especially amid the COVID-19 pandemic, is a challenging case of interest that requires high efforts and effective dedicated plans. In most conditions, drugs induce immunity system weakness and suppression compared to healthy people who are not administering drugs. With the emergence of COVID-19, the severity of drug abuse has increased significantly. Primary care physicians and public health scientists are the frontlines against this burden within the community. The proper investigation includes effective planning for the issues, enumeration for types of abused drugs, significant reasons for widespread of the drugs and planning for effective strategic plans to rehabilitate affected individuals.

\section{Funding: No funding sources \\ Conflict of interest: None declared \\ Ethical approval: Not required}

\section{REFERENCES}

1. Garofoli M. Adolescent substance abuse. Prim Care. 2020;47(2):383-94.

2. Camí J, Farré M. Drug addiction. N Engl J Med. 2003;349(10):975-86.

3. Parasher A. COVID-19: current understanding of its pathophysiology, clinical presentation and treatment. Postgrad Med J. 2021;97(1147):312-20.

4. Sussman S, Skara S, Ames SL. Substance abuse among adolescents. Subst Use Misuse. 2008;43(1213):1802-28.

5. Frances RJ. Substance abuse. JAMA. 1991;265(23):3171-2.

6. Dubey MJ, Ghosh R, Chatterjee S, Biswas P, Chatterjee S, Dubey S. COVID-19 and addiction. Diabetes Metab Syndr. 2020;14(5):817-23.
7. Erol A, Karpyak VM. Sex and gender-related differences in alcohol use and its consequences: contemporary knowledge and future research considerations. Drug Alcohol Depend. 2015;156:113.

8. Meckel KR, Kiraly DD. A potential role for the gut microbiome in substance use disorders. Psychopharmacology (Berl). 2019;236(5):1513-30.

9. Chick J. Alcohol and COVID-19. Alcohol Alcohol. 2020;55(4):341-2.

10. Delirrad M, Mohammadi AB. New methanol poisoning outbreaks in iran following COVID-19 pandemic. Alcohol Alcohol. 2020;55(4):347-48.

11. Mungmungpuntipantip R, Wiwanitkit V. Sharing alcoholic drinks and a COVID-19 outbreak. Alcohol Alcohol. 2020;55(4):343.

12. DeSousa A. Disulfiram ethanol reaction in a patient abstinent from alcohol caused by hand sanitizing. Alcohol Alcohol. 2020;55(4):349.

13. Szabo G, Saha B. Alcohol's effect on host defense. Alcohol Res. 2015;37(2):159-70.

14. Zyl-Smit RN, Richards G, Leone FT. Tobacco smoking and COVID-19 infection. Lancet Respir Med. 2020;8(7):664-5.

15. O'Donnell J, Gladden RM, Mattson CL, Hunter CT, Davis NL. Vital signs: characteristics of drug overdose deaths involving opioids and stimulants 24 states and the district of Columbia, January-June 2019. MMWR Morb Mortal Wkly Rep. 2020;69(35):1189-97.

16. Wainwright JJ, Mikre M, Whitley P, Dawson E, Huskey A, Lukowiak A, et al. Analysis of drug test results before and after the US declaration of a national emergency concerning the COVID-19 outbreak. JAMA. 2020;324(16):1674-7.

17. Ochalek TA, Cumpston KL, Wills BK, Gal TS, Moeller FG. Nonfatal opioid overdoses at an urban emergency department during the COVID-19 pandemic. JAMA. 2020;324(16):1673-4.

18. Pastor F, Folgar MI, Carvalho N, Carvalho F, Horcajadas FA. Therapeutic cannabis and COVID19: between opportunism and infoxication. Adicciones. 2020;32(3):167-72.

19. Laar MW, Oomen PE, Miltenburg CJA, Vercoulen E, Freeman TP, Hall WD. Cannabis and COVID-19: reasons for concern. Front Psychiatry. 2020;11:601653.

20. Khalsa JH, Bunt G, Maggirwar SB, Kottilil S. COVID-19 and cannabidiol (CBD). J Addict Med. 2021;15(5):355-6.

21. Tenegra JC, Leebold B. Substance abuse screening and treatment. primary care. Clinic Office Pract. 2016;43(2):217-27.

Cite this article as: Esmail ME, Mosly MD,

Alghamdi AA, Hakami AH, Majrashi AA,

Abdulrahim SS, et al. Substance abuse, drug addiction and the role of primary health care amid the COVID-19 pandemic. Int J Community Med Public Health $2021 ; 8: 6175-8$. 